\title{
INDICADORES DE RESULTADOS DE LAS PROGRAMAS DE PEDAGOGÍA BÁSICA: EVIDENCIA PARA MEJORAR EL ASEGURAMIENTO DE LA CALIDAD DE LOS DOCENTES EN CHILE ${ }^{1}$
}

\author{
Rosario Rivero ${ }^{2}$ \\ Constanza Hurtado ${ }^{3}$
}

\begin{abstract}
RESUMEN
Los sistemas de acreditación de Formación Inicial Docente (FID) combinan, generalmente, indicadores de insumos y procesos con indicadores de desempeño de sus egresados. Sin embargo, el sistema actual de acreditación de programas de Pedagogía en Chile no contempla evaluaciones de resultados propiamente tales en términos de logro de los propósitos formativos. El contexto internacional, sumado a la creciente exigencia nacional de mejora a la FID, motiva a explorar y expandir los actuales indicadores de calidad disponibles de las instituciones formadoras. En este trabajo se construyen indicadores de empleabilidad y retención de los egresados a nivel de institución y se analiza su varianza y coherencia con el actual sistema de acreditación y con los resultados institucionales en la evaluación docente INICIA.
\end{abstract}

Palabras clave: Formación Inicial Docente, acreditación, primer empleo, carrera profesional, evaluación docente.

\section{PERFORMANCE INDICATORS OF TEACHER TRAINING PROGRAMS: EVIDENCE FOR IMPROVING THE TEACHER QUALITY ASSURANCE SYSTEM IN CHILE}

\section{ABSTRACT}

The accreditation systems for teacher training programs generally combine input/process indicators with output/performance indicators. However, in Chile, the current national accreditation system of teacher training programs does not take into account output/performance indicators. The international context and the growing national demand for improving pre-service teacher education programs encourages us to explore and to expand the current quality indicators present in the teacher training programs. In this paper, indicators for teacher employment and retention rates of new teacher graduates are created and analyzed for their variance and coherence with the current accreditation system and with the institutional results on the teacher evaluation, INICIA.

Keywords: Teaching training programmes, accreditation, first job, professional career, teacher evaluation.

1 Este trabajo contó con apoyo financiero de la Convocatoria CNED 2014 de apoyo a la investigación en educación.

2 Facultad de Educación de la Universidad Diego Portales, Santiago, Chile. Contacto: rosario. rivero@udp.cl

3 Centro de Estudios de Políticas y Prácticas en Educación (CEPPE), Santiago, Chile. Contacto: constanza.hurtado@gmail.com 
18 INDICADORES DE RESUlTADOS DE LAS PROGRAMAS DE PEDAGOGÍA BÁSICA: EVIDENCIA PARA MEJORAR EL ASEGURAMIENTO DE LA CALIDAD DE LOS DOCENTES EN CHILE - R. Rivero y C. Hurtado

\section{Introducción}

El sistema de educación superior en Chile se encuentra en un momento clave respecto de la regulación y el aseguramiento de la calidad, preocupación que data desde hace algunas décadas. El Consejo Superior de Educación de principios de la década de los noventa fue pionero en plantear y promover sistemas de autorregulación de las instituciones de formación superior (Zapata y Tejeda, 2009). A esta iniciativa se sumó el Consejo de Rectores de las Universidades Chilenas (CRUCH) y más adelante el Ministerio de Educación (Mineduc), entidad que tras una consulta pública (1998) planteó una serie de programas, entre ellos los Mecesup ${ }^{4}$, con el propósito de asegurar la consistencia entre lo que las instituciones declaraban acerca de su formación y lo que efectivamente hacían. La promulgación de la Ley de Aseguramiento de la Calidad (ley no 20.129) el año 2006 y la consecuente creación de la Comisión Nacional de Acreditación (CNA) fueron la culminación de un proceso que sentó las bases del sistema que busca asegurar la calidad de los programas de formación docente.

Paralelamente se instaló en el mundo la idea de que la formación docente es uno de los elementos esenciales para mejorar los sistemas de educación (Organisation for Economic Cooperation and Development, 2005). Uno de los componentes de esta perspectiva se refiere al mejoramiento de los sistemas de aseguramiento de la formación docente, en particular, al desarrollo de regulaciones que permitan supervisar la calidad de las instituciones y sus programas (Ingvarson, 2013a). La evidencia muestra que a mayores políticas de aseguramiento -y coherencia entre acreditación de programas, cumplimiento de estándares y certificaciones de los profesores en ejercicio- los egresados demuestran mayor efectividad en el aula (Tatto, Peck, Schwille, Bankov, Senk, Rodríguez y Rowley 2012).

Este escenario ha reforzado la idea de que la selección al comienzo de la formación debe complementarse con evaluaciones

4 Programa del Ministerio de Educación que desde 1998 financia planes de mejoramiento de la educación terciaria. Su foco principal es el fortalecimiento de la planificación estratégica, desarrollo de información para la toma de decisiones basada en indicadores de desempeño (tasas de retención y titulación estudiantil, por ejemplo). Este programa fue antecesor de los Convenios de Desempeño y sentó las bases de una política de aseguramiento orientada a los resultados de aprendizaje y desempeño de las instituciones. 
institucionales como la acreditación. En la actualidad, todos los sistemas de aseguramiento se orientan a los mismos propósitos; controlar, verificar y fomentar la calidad de la educación superior y, a su vez, generar información oportuna para la gestión de las instituciones y para la toma de decisiones de futuros estudiantes y público en general (Zapata y Tejeda, 2009). Para cumplir con ambos objetivos la acreditación debe ser funcional a la dimensión formativa de los programas (apoyo a la planificación institucional, fomento y mejoramiento continuos) y, simultáneamente, a una dimensión de rendición de cuentas de estas (información pública y certificación de calidad, control, direccionamiento del financiamiento) (Zapata y Tejeda, 2009). Esto da fruto a dos dimensiones propias de la acreditación: una de coherencia interna y otra externa.

Si bien desde el año 2007 la acreditación de los programas de Pedagogía en Chile es obligatoria, esta se ha centrado en la dimensión de coherencia interna. La evidencia internacional entrega algunas luces acerca del modo de abordar el vínculo entre la acreditación y los indicadores externos. Ejemplo de ello es el uso de información de la inserción laboral de los egresados, observada en sistemas europeos y norteamericanos, bajo el supuesto de que existe un vínculo entre el sistema de aseguramiento y las competencias laborales desarrolladas por los programas de formación (Tuning Project, 2006; Scharager, Cortés y Bravo, 2008) $)^{5}$.

Por ejemplo, el National Council for Accreditation (NCATE) es una institución especializadas en la acreditación de programas de Pedagogía en Estados Unidos en la que los programas participan voluntariamente del proceso por el status que gozan tras obtener la acreditación de estas agencias. Esta institución se diferencia por el énfasis otorgado a los estándares de capacidades de los egresados versus una mayor centralidad en la coherencia interna y verificación de su cumplimiento por parte de las instituciones (TEAC) (Ingvarson, Elliot, Kleinhenz y McKenzie, 2006). En el proceso de acreditación de NCATE se analiza el cumplimiento de seis estándares, los dos primeros abordan el desempeño de los candidatos (conocimiento y habilidad y, sistema de evaluación) y los cuatro siguientes las capacidades institucionales de los programas (prácticas a lo largo de la formación, diversidad, desarrollo de la facultad y, unidad de recursos y gestión). Para cada uno, existen rúbricas que permiten calificar si el programa presenta un nivel "inaceptable", "aceptable", o "logrado". En el último estándar se usa como criterio el grado de vínculo entre el programa y empleadores de sus egresados, específicamente, se analizan las evaluaciones durante primer año de práctica, la existencia de seguimiento de egresados y reportes de sus empleadores, muestras del trabajo de los candidatos en aula (portafolios), credenciales y experiencia en aula de los docentes de la facultad (Council for the Acreditation of Educator Preparation, 2013). Tras la evaluación, los programas pueden ser acreditados por siete, cinco o dos años, en el último caso se asignan visitas periódicas del comité de NCATE a la institución de las cuales depende la mantención de la acreditación; también es posible que se niegue la acreditación (NCATE, 2010-2014). 
Los estudios de acreditación de FID en Chile se han centrado en aspectos como nivel de exigencia de los criterios, consecuencias, normas y financiamiento (Domínguez y Meckes, 2011); y han señalado la falta de énfasis en criterios vinculados con el mejoramiento de la formación pedagógica, coherencia de las mallas y evaluaciones, inserción temprana de prácticas o referencia a los estándares de formación, entre otras debilidades de la política. Respecto de las consecuencias, se sostiene que no son lo suficientemente persuasivas para lograr el impacto deseado, ya que la no acreditación o pocos años de esta solo se vincula a una restricción de financiamiento y no a medidas como el cierre de los programas (Domínguez y Meckes, 2011).

El presente trabajo busca analizar la coherencia del actual sistema de acreditación con indicadores de tasas de empleabilidad y retención de los egresados de programas de FID y contribuir con nueva evidencia que retroalimente el sistema de acreditación de programas de Pedagogía Básica. Respecto de ello, en la actualidad se sabe que el $85 \%$ y el $88 \%$ de los egresados de Pedagogía Básica se emplean el primer y el segundo año, respectivamente (Portal Mi Futuro, 2015) ${ }^{6}$. En relación con las tasas de retención, Rivero (2015) muestra que el 66\% de los egresados en el año 2007 se mantenía trabajando en el sistema escolar al año 2011, mientras que el 34\% había abandonado el trabajo en escuelas. En la actualidad, se desconoce si los programas con mayor acreditación tienen una mayor/menor inserción laboral de sus egresados y/o una mayor/menor retención de estos en el sistema educacional.

A continuación, se presenta una descripción de los programas de Pedagogía Básica y se revisan las características del sistema de acreditación chileno. Adicionalmente, se presenta evidencia empírica acerca de la relación entre las características de los programas de formación y la inserción laboral de los profesores, su retención y resultados en los exámenes de conocimiento de egreso. Luego, se presentan los objetivos, la metodología de trabajo y los datos

6 Esta proporción se calcula sobre la base de la proporción de titulados de la carrera que obtiene un ingreso superior al mínimo al cabo del primer y segundo año (Portal Mi Futuro, 2015). Se desconoce si estos indicadores provienen de estimaciones a nivel institucional, de las carreras, o bien, del total general de titulados. 
y variables utilizados. Por último, se exponen los resultados y conclusiones de este trabajo.

\section{Contexto de los programas de Pedagogía Básica}

Los programas de Pedagogía Básica han experimentado cambios sustantivos en la última década en Chile. Así lo han descrito investigaciones que destacan el aumento de matrícula de alumnos y la mayor apertura de aquellos poco selectivos por los requisitos que exigen a sus postulantes (Cox, Meckes y Bascopé, 2011). El panorama general de las carreras del área de educación muestra que su promedio de ingreso en la Prueba de Selección Universitaria (PSU) es el más bajo entre las áreas profesionales y que es menor al promedio nacional, e incluso, alrededor de un tercio de quienes ingresan a carreras del área no rinden la PSU (Eyzaguirre e Inostroza, 2014). Adicionalmente, las carreras de Pedagogía Básica han experimentado un descenso en los puntajes desde 500 puntos promedio en el 2012 a 486 puntos en 2015 (Consejo Nacional de Educación, 2015). Asimismo, se registra una caída en el número de estudiantes que ingresan a estudiarla: el año 2015 la matrícula de primer año fue de 1.872 alumnos, cifra que representa un descenso del $71 \%$ si se compara con aquellos alumnos que ingresaron en el año 2005 (CNED, 2015).

Coherentemente con la evaluación de los matriculados, el número de titulados también presenta una tendencia a la baja. En el periodo 2008 a 2013, se observa una disminución de 38\%, desde 5.538 a 3.407 alumnos. Aun así, la cantidad de instituciones con alumnos titulados aumentó de 47 en el 2008, a 55 en el 2013 (Fuente: SIES 2015). Al observar las características institucionales, destaca un incremento de los egresados carreras de institutos profesionales e instituciones privadas. Específicamente, en el año 2008 un 9\% del total de titulados provenía de universidades privadas, mientras que para el año 2013 este había aumentado a un 15\%. Por su parte, se observa una disminución del $75 \%$ de titulados de las carreras impartidas por universidades estatales del CRUCH. Entre el año 2008 y 2013 disminuyeron en 26 las carreras de Pedagogía en este tipo de instituciones. 
En relación con los indicadores de calidad disponibles para estos programas, las cifras muestran que en el año 2015 un 65\% (88 de 136) de las carreras de Pedagogía Básica cuenta con acreditación, proporción similar a lo observado en el año 2008, cuando los programas acreditados correspondían a un 60\% (CNED, 2015). Las carreras de Pedagogía están acreditadas en 4,3 años promedio, siendo este valor más alto para carreras impartidas en universidades del CRUCH (4,7 años promedio) al compararlas con universidades privadas (4 años promedio) e institutos profesionales (3,4 años promedio) (Centro de Estudios de Políticas y Prácticas en Educación, 2013).

Un segundo indicador disponible corresponde a los resultados en la prueba INICIA. Aun cuando la prueba es voluntaria sus resultados revelan un bajo dominio de los contenidos disciplinarios y pedagógicos necesarios para ejercer la profesión. El porcentaje promedio de preguntas correctas en las pruebas de conocimientos disciplinarios y pedagógicos se han situado en torno al 50\% en los años de rendición. También se ha destacado una alta heterogeneidad de resultados entre las instituciones, existiendo casos en donde todos los egresados obtuvieron resultados en las categorías "Aceptable" o "Sobresaliente" en la prueba de conocimientos disciplinarios de 2011, mientras que en otras instituciones más de un $60 \%$ se concentra en la categoría "Insuficiente".

\section{Revisión bibliográfica}

\subsection{Descripción del sistema de acreditación de programas de FID en Chile}

Desde el año 2007, la acreditación de carreras conducentes al título profesional de profesor o educador es obligatoria y prerrequisito para recibir financiamiento estatal, quedando la entrega del Crédito con Aval del Estado, y una serie de becas ofrecidas por este, condicionados a la situación de acreditación del programa y/o institución donde los estudiantes se matriculan. El propósito principal de este procedimiento es certificar la calidad de las carreras ofrecidas por las instituciones en función de los propósitos declarados por estas y 
los señalados en los estándares nacionales e internacionales de cada profesión (ley no 20.129). La Comisión Nacional de Acreditación establece nueve criterios de evaluación que definen las expectativas del funcionamiento de los programas de Pedagogía, estos se agrupan en tres dimensiones. Para cada criterio se distinguen aspectos que los programas deben cumplir de aquellos que deberían cumplir ${ }^{7}$. El proceso consta de una evaluación interna y una externa a cargo de una agencia autorizada por la Comisión Nacional de Acreditación (CNA) (ley $n^{\circ}$ 20.129). Complementariamente, el análisis incluye a todos los programas impartidos por una misma institución conducente a un título profesional equivalente (por ejemplo, todos los programas que otorguen el título de profesor de Educación Básica). La única distinción se refiere a procesos de acreditación de instituciones que cuentan con cohortes de egresados de aquellas que no ${ }^{8}$ los tienen.

La primera dimensión evaluada es el Perfil de egreso y resultados. En esta se incluyen cuatro subdimensiones: perfil profesional del educador, características de la malla curricular, resultados del proceso de formación y vinculación con el medio. Entre los criterios usados para evaluar esta dimensión se exige que el perfil considere el análisis y comprensión del ejercicio de la profesión y el desarrollo de capacidades propias de un profesional. A su vez, los programas de estudios tienen que incluir las siguientes cuatro áreas de formación: general, especialidad, formación profesional (por ejemplo, procesos de enseñanza y cognitivos) y formación práctica (al menos un semestre de experiencia práctica). Luego en la subdimensión de resultados del proceso de la formación, se analiza la existencia de mecanismos de seguimiento de los procesos académicos, esto incluye tasas de retención, aprobación, titulación y tiempo de egreso, entre otros. En particular, se debe demostrar la existencia de indicadores de eficiencia de la enseñanza, análisis de causas de deserción de los estudiantes y procesos de seguimiento de los egresados, para

7 En la ley no 20.129 se establece que hay elementos que son obligatorios y otros voluntarios de demostrar en los procesos de acreditación.

8 Tanto la estructura, dimensiones y criterios de acreditación son equivalentes para programas de Educación Parvularia, Educación Diferencial, Pedagogía Básica y Pedagogía Media (CNA, 2015). Según el desempeño de cada institución el resultado de la acreditación otorga un plazo de acreditación que varía entre uno hasta siete años, o de lo contrario no se acredita la carrera. 
24 INDICADORES DE RESUlTADOS DE LAS PROGRAMAS DE PEDAGOGÍA BÁSICA: EVIDENCIA PARA MEJORAR EL ASEGURAMIENTO DE LA CALIDAD DE LOS DOCENTES EN CHILE - R. Rivero y C. Hurtado

actualizar y perfeccionar los planes y programas de estudio. En la última subdimensión se observa la relación con el medio externo, constatable por medio de actividades de extensión, actividades de servicios a terceros, participación en discusiones nacionales y de la región, entre otras (CNA, 2015).

La segunda dimensión evaluada corresponde a las Condiciones mínimas de operación, en la que se analizan las subdimensiones la estructura organizacional, administrativa y financiera, recursos humanos, efectividad del proceso de enseñanza-aprendizaje e infraestructura y recursos para el aprendizaje. Para evaluar estos ámbitos se aplican criterios de eficacia de la gestión financiera, de evaluación de las características del cuerpo directivo, metodologías de selección de funcionarios y pertinencia de los mecanismos de admisión de los estudiantes según el diseño de cada institución y adecuación de la infraestructura de la unidad, entre otros.

Por último, en la tercera dimensión, llamada Capacidad de autorregulación, se contemplan como subdimensiones los propósitos del programa, integridad y autoevaluación. Esto se evalúa por medio de criterios de existencia de postítulos o posgrados para los egresados, coherencia entre los propósitos definidos, las asignaturas que componen el plan de estudio y los resultados que obtienen los alumnos, entre otros (CNA, 2015).

Si bien la primera dimensión de la acreditación alude a resultados, no contempla evaluaciones en términos del logro de los propósitos formativos. En lo relativo a la empleabilidad de los egresados se señala que las instituciones deben hacer seguimiento de ello, sin embargo, no se explicita la inclusión de tasas de empleabilidad ni de retención, sino que más bien se trata de ejemplos de información que debiese estar disponible en las instituciones. Es decir, es voluntario hacer seguimiento a los egresados, no se encuentran estándares a nivel nacional como porcentajes mínimos relacionados con las tasas de empleabilidad y retención de las cohortes de egresados, ni referencias y orientaciones acerca de cómo calcular estos indicadores, su significado y potenciales usos internos y externos. Esto no significa que no existan instituciones que generen esta información para los procesos de acreditación, pero 
sí que aquellas que lo hacen lo realizan de forma voluntaria o debido a un compromiso realizado en los convenios de desempeño.

2.2. Evidencia de características de programas de formación relacionadas con empleabilidad y resultados de los egresados

\subsubsection{Internacional}

El vínculo entre los sistemas de aseguramiento y el mercado laboral docente depende de algunos elementos, entre ellos si la información de acreditación es pública o si es obligatorio estudiar en instituciones acreditadas para trabajar en una escuela, como ocurre en algunos estados en Estados Unidos (Council for Higher Education Accreditation, 2010).

Acerca de las características de los sistemas de aseguramiento y resultados de los egresados, el estudio TEDS-M (Tatto et al., 2012) analiza el resultado en pruebas que miden habilidades y conocimiento para la enseñanza de la Matemática de futuros docentes provenientes de 17 países y de 500 instituciones formadoras; se encuentra una alta varianza en términos de conocimiento de los docentes al interior de los países. Sin embargo, es posible distinguir políticas que parecen más efectivas en el logro de aprendizajes de egresados a la luz de los resultados promedio, ya que existen diferencias que favorecen a profesores formados en sistemas con estándares de acreditación más exigentes, versus los resultados de aquellos que presentan políticas más débiles en este ámbito y con consecuencias más laxas para las instituciones que no cumplen con los estándares determinados (en este último grupo se incluye Georgia y Chile) (Ingvarson, et. al, 2011). Asimismo, los sistemas de aseguramiento de la calidad más efectivos incluyen exigencias de ingreso y cualificación de los programas de estudio y se caracterizan por una coordinación entre las políticas de aseguramiento de la formación docente. Esto se traduce en diseños que contemplan desde el reclutamiento hasta sistemas de regulación basados en la revisión constante, evaluación y acreditación de los programas (Tatto, 2012).

También se ha explorado la relación entre las características de los programas y las trayectorias laborales de los docentes en 
estudios que consideran el efecto a nivel individual y no agregado. Investigaciones longitudinales de egresados, por su parte, muestran que si bien la FID se vincula con las trayectorias laborales (una mayor preparación en la formación para trabajo con alumnos con necesidades educativas especiales se relaciona positivamente con la permanencia en escuelas con estudiantes de estas características) el efecto es menor que el nivel socioeconómico de los docentes y que aspectos subjetivos, como su motivación por enseñar (Kirchhoff y Lawrenz, 2011; Freedman y Appleman, 2009). Luego, programas de corta duración (un año y prácticas intensivas) se relacionan positivamente con la permanencia de los docentes jóvenes en escuelas urbanas (Jorissen, 2003). Asimismo, se ha observado que el contexto institucional de los programas de formación -el vínculo con escuelas donde se realizan las prácticas- y las percepciones de los egresados acerca de los distintos tipos de establecimientos, influyen en sus postulaciones y elecciones al momento de empezar a ejercer (Maier y Youngs, 2009). Redes más sólidas posibilitan mayor información y colaboración entre docentes novatos y aquellos experimentados, así como las inducciones serían un factor preventivo de la deserción temprana de la profesión docente (Liu y Johnson, 2006). Esto se explica por la percepción de alta responsabilidad y escaso apoyo que perciben quienes ingresan a trabajar sin experiencias de práctica. En esta línea, es manifiesto que la debilidad en la formación de los docentes para ejercer en escuelas de alumnos más vulnerables (en términos de prácticas o mentorías en estos contextos) es uno de los factores explicativos de la menor postulación de los egresados a este tipo de escuelas (Boyd, Lankford, Loeb y Wyckoff 2005). Luego, la ubicación de las instituciones donde los docentes cursaron sus estudios afecta -en menor grado que su ubicación geográfica de origen- el lugar donde comienzan a ejercer, siendo esto más evidente en el caso de instituciones ubicadas en sectores urbanos donde los estudiantes se trasladan para cursar los programas (Boyd et al., 2003).

\subsubsection{Nacional}

La investigación nacional acerca del vínculo entre el sistema de aseguramiento y la empleabilidad o resultados en evaluaciones de los egresados es escasa. Existe evidencia de la relación entre los años 
de acreditación con las reacciones institucionales frente a la prueba INICIA, encontrándose que carreras con más años de acreditación presentan reacciones positivas y orgánicas ante esta medida, al comparar con instituciones de menor acreditación, para las que se registra mayor presión en profesores y estudiantes y estrategias instrumentales para aumentar los puntajes (Meckes, Taut, Bascopé, Valencia y Manzi, 2012). Un trabajo pionero respecto de la relación entre la acreditación y los resultados de los egresados es el desarrollado por Domínguez, Bascopé, Meckes y San Martín (2012); en este se estudia la relación entre años de acreditación de los programas y resultados en prueba INICIA, encontrándose una débil y errática relación entre ambos indicadores.

Existe mayor evidencia en torno a los patrones de inserción en las escuelas de los docentes nóveles según características de la formación (duración, selectividad de las instituciones), siendo claro el ordenamiento entre las características de los egresados y de los alumnos a los que enseñan (Ruffinelli y Guerrero, 2009; Meckes y Bascopé, 2010; Rivero, 2015). Así, docentes con mayores puntajes en las pruebas de selección universitaria inician su carrera en escuelas de alto nivel socioeconómico con mayor probabilidad (Cabezas, Gallegos, Santelices y Zarhi, 2011) y docentes de peores resultados en la evaluación INICIA presentan mayor probabilidad de insertarse en escuelas de más bajo nivel socioeconómico (Meckes y Bascopé, 2012). Complementariamente, la evidencia muestra que docentes que cursaron sus programas de formación en jornada diurna, se integran en mayor proporción a escuelas particulares subvencionadas que a escuelas municipales. También, que quienes comienzan a ejercer en escuelas de menor nivel socioeconómico declaran que en sus programas de formación se les impulsó a hacerlo; por el contrario, docentes de altos puntajes en la PSU declaran una mayor motivación durante su formación a trabajar en escuelas privadas. En términos de las prácticas profesionales, su asignación por criterio de cercanía al hogar de los estudiantes, fomenta su posterior inserción en escuelas de características similares a aquellas en que los docentes cursaron sus estudios escolares. Docentes que realizaron su práctica en establecimientos particulares subvencionados se insertan con menor probabilidad en escuelas municipales (Paredes, Bogolasky, 
Cabezas, Rivero y Zahri 2013). Este ordenamiento tendría su origen en el proceso de búsqueda del primer trabajo respecto de lo cual se ha constatado que los egresados de instituciones con más años de acreditación tienen más oportunidades de postular a establecimientos particulares pagados, en tanto que los egresados de institutos profesionales o de centros de formación técnica presentan mayores probabilidades de postular a escuelas de dependencia municipal (Flores, Bogolasky, Cabezas, Rivero y Zahri, 2013). Por su parte, el tipo de escuelas en las que los docentes comienzan a trabajar determina su trayectoria posterior, existiendo una alta relación entre la dependencia administrativa del primer empleo y su permanencia en estos a lo largo de su trayectoria laboral (Paredes et al., 2013). En esta línea, también se ha observado que características como la acreditación de la institución formadora se relaciona con los cambios a lo largo de la trayectoria laboral, ya que los docentes de instituciones acreditadas migran a establecimientos educacionales de mayor nivel socioeconómico (Cabezas et al., 2011).

\section{Objetivos}

El propósito general del estudio es explorar y expandir los actuales indicadores de calidad de las carreras de Pedagogía Básica hacia la evaluación del desempeño de los egresados en el ámbito laboral. Los objetivos específicos son:

a. Desarrollar indicadores de programas de Pedagogía que evalúen el desempeño de los egresados en el ámbito laboral (empleabilidad y retención).

b. Analizar el desempeño de los programas de Pedagogía Básica en Chile sobre la base de los distintos indicadores de empleabilidad y retención por institución, según su varianza (entre instituciones y dentro de estas).

c. Estudiar la coherencia entre estos nuevos indicadores de desempeño de los egresados y los años de acreditación de los programas de Pedagogía y el desempeño de los egresados en la prueba INICIA.

d. Evaluar el uso de estos indicadores para orientar la toma de decisiones de políticas públicas que apunten a mejorar 
el sistema actual de acreditación y de aseguramiento de la calidad de formación de los docentes en Chile.

\section{Metodología}

La metodología de este estudio cuenta con tres fases. Con el objetivo de conocer el comportamiento de los indicadores de empleabilidad y retención, en la primera fase se analizan los resultados descriptivos de estos, estimados a nivel de carrera y por cohorte de titulados. Para estudiar la asociación entre características institucionales y de las carreras con los indicadores, en la segunda fase se descompone la varianza de estos indicadores a nivel de carrera ${ }^{9}$ en: la proporción de varianza entre instituciones y la proporción de varianza entre carreras dentro de una misma institución. También se estudia la descomposición de la varianza de los indicadores de empleabilidad y retención según variables institucionales y de las carreras, por año.

Para descomponer la varianza se usa un análisis de varianza basado en ANOVA unidireccional (oneway). Para cada nivel, $\eta^{2}$ es la proporción de varianza entre grupos; $\eta^{2}$ es la razón entre la suma de los cuadrados entre grupos ( $\mathcal{S}$ Between) y la suma total de cuadrados ( $S S T$ total). La ecuación (1) muestra este cálculo.

$n^{2}=\frac{\text { SS Between }}{\text { SSTotal }}=\frac{\sum\left(x_{j}-\bar{x}\right)^{2}}{\sum\left(x_{i j}-\bar{x}\right)^{2}} n^{2}=\frac{\text { SS Between }}{\text { SSTotal }}=\frac{\sum\left(x_{j}-\bar{x}\right)^{2}}{\sum\left(x_{i j}-\bar{x}\right)^{2}}(1)$

Donde:

$x_{j}=$ promedio grupo $j$

$\bar{x}=$ media total

$x_{i j}=$ indicador empleabilidad carrera $i$ en grupo $j$

Con el objetivo de explorar la relación entre las características de las instituciones y las carreras, la tercera fase consiste en estimar

9 Se entiende por carrera un programa de Pedagogía Básica impartido en una institución, en una sede determinada y en un horario determinado (vespertino, diurno u otro). Se usan las carreras como unidad, ya que en este nivel se distinguen distintas sedes de una misma institución, variable que puede incidir en la empleabilidad de los titulados al igual que el horario en el que se estudió la carrera. 
30 INDICADORES DE RESUlTADOS DE LAS PROGRAMAS DE PEDAGOGÍA BÁSICA: EVIDENCIA PARA MEJORAR EL ASEGURAMIENTO DE LA CALIDAD DE LOS DOCENTES EN CHILE - R. Rivero y C. Hurtado

regresiones para analizar si los años de acreditación de los programas de Pedagogía se asocian con los indicadores de empleabilidad y retención ${ }^{10}$. Se estiman modelos jerárquicos en donde las carreras se encuentran anidadas en instituciones, la ecuación (2) muestra que en un primer nivel se incluyen variables de las carreras y en el segundo nivel variables institucionales.

Indicador $_{i j}=\beta_{0 i}+$ Características institucionales $_{j}+{\text { Características } \text { Carrera }_{i j}}$ + Variables Contexto $_{i j}+\theta_{i j}(2)$

Donde:

Indicador $_{i j}=$

Indicador de empleabilidad o retención de carrera i en institución $j$ Características insti caracteristicas de instituciónj

Características Carrera $a_{i j}=$ características de la carrera $i$ en institución $j$

Variables Contexto $_{i j}=$ variables de contexto de carrera $i$ en institución $j$

$\theta_{i j}=$ error

4.1. Datos, muestra y variables

4.1.1. Datos

El universo de este estudio corresponde a 21.938 profesores titulados de todos los programas de Pedagogía Básica del país entre los años 2008 a 2011. Los datos son individuales y las fuentes son: base de titulados de programas de Pedagogía Básica 2008- 2011 (SIES), base de idoneidad docente 2008 a 2014 (Mineduc) y bases de resultados de la prueba INICIA 2008-2012 (Mineduc). Complementariamente, se usan bases de datos a nivel de carreras e instituciones, estas son: bases de acreditación históricas desde el año 2003 al 2014 (CNA), base de titulados por carrera 2005-2012 (SIES) y base institucional histórica 2005-2012 (CNED).

A partir de los datos de carreras se construyó una base anual (2008-2011) de carreras de Pedagogía Básica a la cual se asociaron datos acerca del tipo de institución a la que pertenece la carrera,

10 Se usa como unidad la carrera, ya que los análisis de varianza muestran que la varianza entre instituciones explica una proporción importante de la varianza de los indicadores de empleabilidad y retención, al igual que el año de la cohorte. 
región en la que se imparte, el total de titulados de la carrera, los años de acreditación de la carrera, el horario en el que se imparte y la duración en semestres. A estos datos se agregaron los datos individuales de titulados y de idoneidad docente de los años 2008 a 2014, para identificar quiénes están trabajando, y también sus resultados de la prueba INICIA ${ }^{11}$. La Tabla 1 presenta la cantidad de instituciones, carreras y titulados por año. Se observa que alrededor de 50 instituciones ofrecían Pedagogía Básica, las cuales impartían 185 carreras en 2008 y 210 en 2011. Al analizar el número de titulados por institución se observa una alta concentración de carreras en las diez instituciones de mayor tamaño las que forman alrededor del 60\% de los profesionales titulados por año.

Tabla 1: Número de instituciones, programas y titulados de carreras de Pedagogía Básica 2008 a 2011

\begin{tabular}{|c|c|c|c|c|c|}
\hline & \multicolumn{4}{|c|}{ Año cohorte titulados } & \multirow[b]{2}{*}{$\begin{array}{l}\text { Variación } \\
\text { 2011-2008 }\end{array}$} \\
\hline & 2008 & 2009 & 2010 & 2011 & \\
\hline $\mathrm{N}$ instituciones & 47 & 50 & 50 & 50 & 3 \\
\hline N programas & 185 & 210 & 195 & 184 & -1 \\
\hline Titulados & 5538 & 6056 & 5741 & 4603 & -935 \\
\hline 10 instituciones más grandes concentran & \multirow{2}{*}{$63 \%$} & \multirow{2}{*}{$62 \%$} & \multirow{2}{*}{$58 \%$} & \multirow{2}{*}{$61 \%$} & \multirow{2}{*}{$-2 \%$} \\
\hline (\% alumnos titulados) & & & & & \\
\hline 10 instituciones más chicas concentran & \multirow{2}{*}{$5 \%$} & \multirow{2}{*}{$3 \%$} & \multirow{2}{*}{$3 \%$} & \multirow{2}{*}{$3 \%$} & \multirow{2}{*}{$-2 \%$} \\
\hline (\% alumnos titulados) & & & & & \\
\hline
\end{tabular}

Fuente: Elaboración propia.

\subsubsection{Variables}

A partir de las bases de datos se construyeron los indicadores de empleabilidad y retención. En ambos se considera que un alumno titulado está "empleado" si aparece en alguna de las bases de idoneidad

11 La prueba INICIA fue creada el año 2008 con el objetivo inicial de fomentar y apoyar procesos de transformación dentro de las instituciones a través de información de los conocimientos de los egresados de sus programas. En la actualidad, su uso central es informar del nivel de logro de los futuros profesores, además de a las instituciones, a la opinión pública y a los propios egresados de pedagogía. Se evalúan conocimientos y habilidades básicas en el ámbito disciplinario y pedagógico de egresados de programas de formación para los distintos niveles del sistema escolar: parvulario, primaria y secundaria. 
32 INDICADORES DE RESUlTADOS DE LAS PROGRAMAS DE PEDAGOGÍA BÁSICA: EVIDENCIA PARA MEJORAR EL ASEGURAMIENTO DE LA CALIDAD DE LOS DOCENTES EN CHILE - R. Rivero y C. Hurtado

docente, independiente de las horas de contrato, el sueldo percibido, el tipo de contrato, número de establecimientos en que trabaja y su función en las escuelas ${ }^{12}$. Los indicadores se construyeron según la proporción de egresados de cada carrera que se encontraba trabajando en los años consecutivos a la titulación. El indicador de empleabilidad consiste en la proporción de titulados de la carrera que se encontraba trabajando el año de titulación o los dos siguientes ${ }^{13}$. El segundo indicador, retención, se calculó considerando la proporción de egresados que trabajaba el cuarto, quinto o sexto año a partir de la titulación (contando como primer año el de titulación) ${ }^{14}$.

Debido a que las bases de acreditación de carreras de Pedagogía presentaban algunos vacíos entre los años 2008 a 2011 (instituciones sin información de acreditación para la carrera de Pedagogía Básica), la variable de años de acreditación se construyó considerando la información disponible para el año de titulación y se completó con la correspondiente a alguno de los tres años de referencia del indicador de empleabilidad y con información disponible en la base de CNED (indicadores institucionales 2005 a 2015) y en la página web de la $\mathrm{CNA}^{15}$. El valor 0 significa que se rechazó la acreditación. La Tabla 2 presenta la distribución de la variable según año, se observa que la mayor proporción de carreras se concentra en tres años de acreditación (alrededor de 35\% en cada año). La suma de carreras con dos y tres años de acreditación supera el 60\%. También se asoció a las carreras el promedio de la prueba INICIA ${ }^{16}$.

12 Se incluyen todos los casos, ya sea que los titulados se desempeñen como docentes de aula, planta técnico-pedagógica, planta directiva, director(a)/profesor encargado, otra en el establecimiento, otra fuera del establecimiento, jefe unidad técnico-pedagógica, inspector general $u$ orientador (Mineduc).

13 Se observó que en todos los años estudiados más de la mitad de los titulados se encontraba trabajando en el sistema escolar el año de su titulación.

14 Este segundo indicador no se estimó para el año 2011, ya que los datos de empleo en el sistema escolar existen hasta el año 2014

15 Si había una carrera sin información de años de acreditación se usó la correspondiente a la base de CNED para señalar si estaba no acreditada.

16 Se estimó el promedio institucional de puntajes de la prueba INICIA; solo se usan los datos de la prueba disciplinaria rendida entre los años 2008 y 2010, porque mantienen la misma escala de puntaje de respuestas (\% de correctas). El promedio de respuestas correctas se ubica en torno a 50\% en todos los años. 
Tabla 2: Años de acreditación para carreras de pedagogía básica 2008 - 2011

\begin{tabular}{l|c|c|c|c|c|c|c}
\hline & \multicolumn{7}{|c}{ Años acreditación } \\
\hline Año cohorte & 0 & 2 & 3 & 4 & 5 & 6 & Total \\
\hline \multirow{2}{*}{2008} & 17 & 50 & 59 & 31 & 12 & & 169 \\
\cline { 2 - 9 } & $16 \%$ & $29 \%$ & $31 \%$ & $18 \%$ & $5 \%$ & & \\
\hline \multirow{2}{*}{2009} & 32 & 57 & 62 & 36 & 10 & & 197 \\
\cline { 2 - 8 } & $18 \%$ & $23 \%$ & $35 \%$ & $19 \%$ & $3 \%$ & $2 \%$ & \\
\hline \multirow{2}{*}{2010} & 34 & 43 & 65 & 36 & 6 & 3 & 187 \\
\hline \multirow{2}{*}{2011} & $19 \%$ & $17 \%$ & $33 \%$ & $26 \%$ & $3 \%$ & $2 \%$ & \\
\hline \multirow{2}{*}{ Total } & 35 & 30 & 60 & 47 & 5 & 3 & 180 \\
\cline { 2 - 8 } & $19 \%$ & $17 \%$ & $33 \%$ & $26 \%$ & $3 \%$ & $2 \%$ & \\
\hline
\end{tabular}

Fuente: Elaboración propia

Otro conjunto de variables, institucionales y de las carreras, proviene de las bases anuales de titulados de SIES. En particular se usó la información del tipo de institución al que pertenecían las carreras (CRUCH, privadas CRUCH, privadas, institutos profesionales), horario en que se imparte la carrera (diurno, vespertino u otro) y duración de la carrera (ocho semestres, más de ocho semestres). Asimismo, se construyó una variable de selectividad que distingue si el puntaje de corte promedio en la PSU de matemática y lenguaje es inferior a 500 puntos, superior o bien no se registra esta información en la base CNED. Por último, se asoció la variable región en que se imparte la carrera.

Respecto del tipo de institución al que pertenece la carrera sobresale que las universidades privadas concentran la mayor cantidad de casos (50\%), a estas le siguen las carreras de las universidades estatales del CRUCH donde se imparte un tercio de las carreras de Pedagogía Básica. En términos de puntajes de la PSU, se observa que la mayor concentración de carreras se encuentra en la categoría de menos o igual a 500 puntos, las otras dos alternativas -carreras que no solicitan puntajes o no los registran y puntajes de corte superiores a 500 puntos- reúnen a un 25\% de las carreras por año. La mayoría de las carreras se imparte en horario diurno y, en términos de su duración, se observa un cambio desde el año 2008 -cuando la mayoría se extendía por menos de ocho semestres- hasta el año 2011 cuando se distribuyen equitativamente en ambas categorías. 
34 INDICADORES DE RESUlTADOS DE LAS PROGRAMAS DE PEDAGOGÍA BÁSICA: EVIDENCIA PARA MEJORAR EL ASEGURAMIENTO DE LA CALIDAD DE LOS DOCENTES EN CHILE - R. Rivero y C. Hurtado

\section{Resultados}

\subsection{Fase 1: Análisis descriptivo}

La Tabla 3 presenta datos descriptivos de los indicadores de empleabilidad y retención; se exponen los promedios ponderados según el número de matrícula a nivel nacional para cada cohorte de 2008 a $2011^{17}$; los resultados son similares y cercanos a $80 \%$ de empleabilidad en cada año. En relación con el indicador de retención, el porcentaje de titulados empleados a nivel nacional disminuye sustantivamente al comparar con la empleabilidad, ya que hay alrededor de $40 \%$ de retención en cada cohorte ${ }^{18}$. En ambos indicadores la tendencia ha sido a la baja en alrededor de diez puntos porcentuales; se desconoce la razón de la caída del año 2011 en el indicador de empleabilidad. Así, mientras en el año 2008 la empleabilidad de egresados era de 82\%, en el año 2011 era de $73 \%$. En el caso de la retención, la cifra de titulados que trabajaban a partir del cuarto año era de $40 \%$ y en el año 2010 de 32\%. En el caso del indicador de retención cabe destacar que la cantidad años considerados para estimarlo en 2010 es menor que la cantidad considerada para los otros dos años en que se calculó (2008 y 2009), lo que podría explicar la caída en el año 2010.

Tabla 3: Indicador empleabilidad y retención 2008-2011*

\begin{tabular}{l|c|c|c|c|c}
\hline & 2008 & 2009 & 2010 & 2011 & Variación 2011/0-2008 \\
\hline Indicador empleabilidad & $82 \%$ & $81 \%$ & $82 \%$ & $73 \%$ & $-9 \%$ \\
\hline Indicador retención & $40 \%$ & $39 \%$ & $32 \%$ & & $-8 \%$ \\
\hline
\end{tabular}

Fuente: Elaboración propia.

Nota (*): Estos resultados se ponderaron por la cantidad de titulados de instituciones y carreras.

La Tabla 4 muestra los estadísticos descriptivos de los indicadores a nivel institucional y de carrera. Se observa que, en promedio, el $81 \%$ de los titulados se emplea durante los primeros

17 También se calcularon los promedios nacionales de los indicadores de empleabilidad y de retención promediando los resultados obtenidos a nivel institucional y de carreras, y ponderando según la cantidad de titulados. Los resultados son similares a los observados a nivel nacional.

18 Esto es consistente con estudios de empleabilidad de docentes, que destacan la alta deserción de estos profesionales a partir del quinto año de ejercicio. 
tres años a contar de la titulación a nivel de institución, con una desviación estándar de 0,14. Luego, a nivel de carreras, el promedio de empleabilidad es de 79\%; hay casos de carreras en que ninguno de sus titulados se emplea durante los primeros tres años ${ }^{19}$. En el caso de la retención, los promedios son similares a nivel de institución y carrera y muestran que la tasa promedio es de alrededor de un $40 \%$. Se observa que a nivel de institución, el máximo de porcentaje corresponde a un $67 \%$ de los titulados trabajando en el cuarto, quinto o sexto año a partir de la titulación ${ }^{20}$. La correlación entre los indicadores de empleabilidad y retención de las carreras es de 0,34, y es significativa a un nivel de confianza de 0,05.

Tabla 4: Descriptivos de indicadores de empleabilidad a nivel institucional y de carreras 2008-2011

\begin{tabular}{l|l|c|c|c|c|c|c}
\hline Unidad & Variable & Promedio & $\begin{array}{c}\text { Desviación } \\
\text { Estándar }\end{array}$ & Varianza & Min. & Máx. & $\begin{array}{c}\text { N } \\
\text { carreras }\end{array}$ \\
\hline \multirow{3}{*}{ Institucional } & Indicador empleabilidad & 0,78 & 0,15 & 0,02 & 0,28 & 1 & 190 \\
\cline { 2 - 8 } & Indicador retención & 0,35 & 0,13 & 0 & 0 & 0,71 & 142 \\
\hline \multirow{2}{*}{ Carrera } & Indicador empleabilidad & 0,79 & 0,23 & 0,02 & 0 & 1 & 774 \\
\cline { 2 - 8 } & Indicador retención & 0.38 & 0.23 & 0.05 & 0 & 1.00 & 590 \\
\hline
\end{tabular}

Fuente: Elaboración propia.

En la Tabla 5 se presenta la distribución de las carreras según cuartiles de distribución de la variable de empleabilidad. El promedio de empleabilidad del primer cuartil es de $49 \%$, del segundo es de $80 \%$, los últimos dos muestran promedios cercanos al $90 \%$ de empleabilidad. De la Tabla 6 -distribución en cuartiles de la variable de retención- se desprende que el promedio del primer cuartil es aproximadamente $12 \%$ de retención en los tres años; esta cifra asciende a más del doble en el segundo cuartil (35\% de retención) y alcanza alrededor de un 60\% en el último cuartil de distribución de la variable. También se observa el promedio de titulados según cuartiles de los indicadores; en el caso de la empleabilidad los promedios son

19 La cantidad de carreras, en todo el periodo, con $0 \%$ empleabilidad es 22 y con empleabilidad del $100 \%$ es 213.

20 La cantidad de carreras con valor 0 en el indicador de retención es 50 y con retención de un $100 \%$ es de 33 ; estas se distribuyen de manera similar entre años 
similares entre cuartiles, con excepción del segundo cuartil, que reúne a carreras con mayor promedio de titulados. En el caso de la retención los promedios de titulados también son similares y se observan mayores diferencias entre años.

Por último, en las Tablas 7 y 8 se muestra el porcentaje promedio de empleabilidad y retención, según años de acreditación por año y para toda la muestra. Los resultados señalan que las carreras con cinco y seis años de acreditación presentan tasas de empleabilidad promedio menores a las observadas para otros años de acreditación; sin embargo, esta no es la tendencia por año. Luego, en el caso de la retención promedio, la mayor proporción de egresados que se mantiene trabajando se reporta para las carreras con dos años de acreditación; este grupo supera en alrededor de diez puntos porcentuales a los promedios observados para los otros años de acreditación, lo que varía al comparar entre años. 


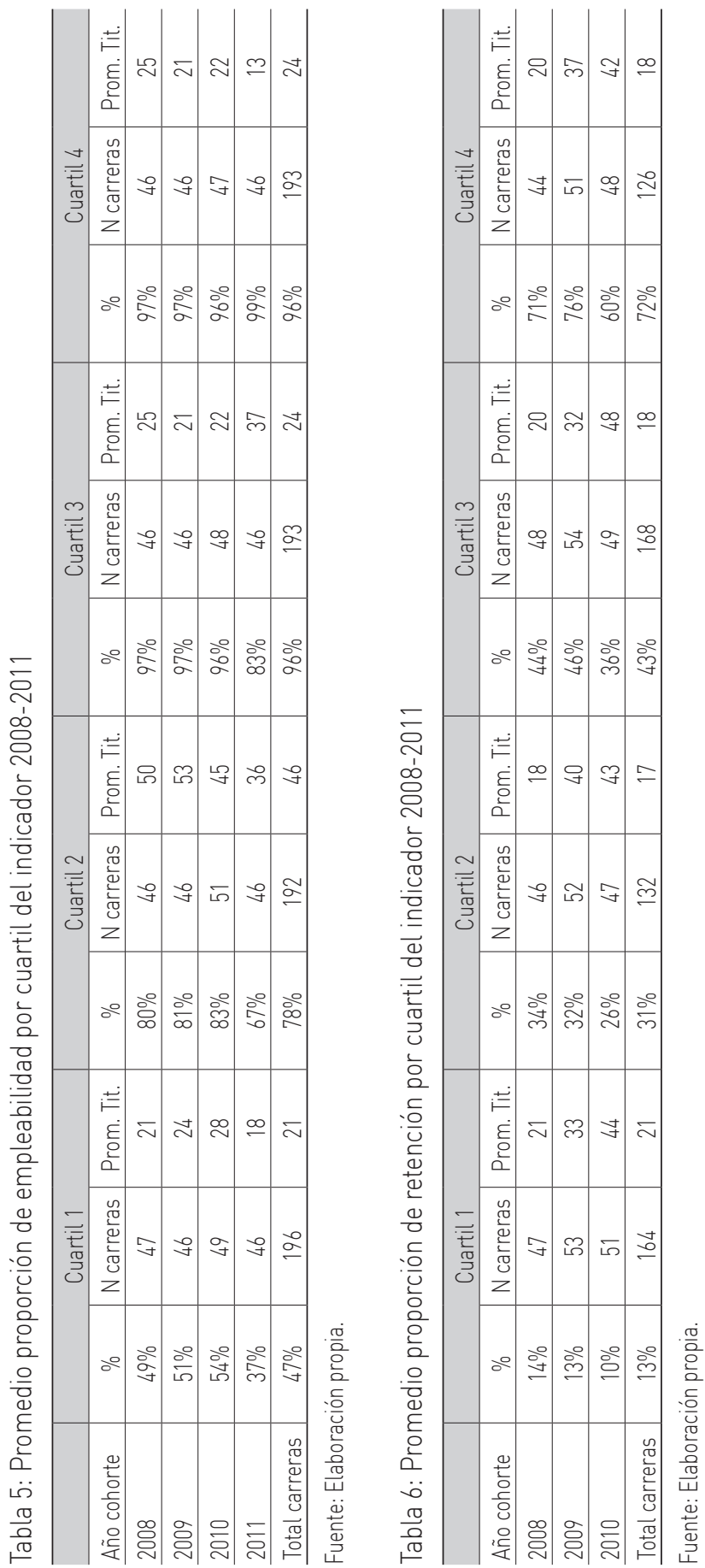


38 INDICADORES DE RESULTADOS DE LAS PROGRAMAS DE PEDAGOGÍA BÁSICA: EVIDENCIA PARA MEJORAR EL ASEGURAMIENTO DE LA CALIDAD DE LOS DOCENTES EN CHILE - R. Rivero y C. Hurtado

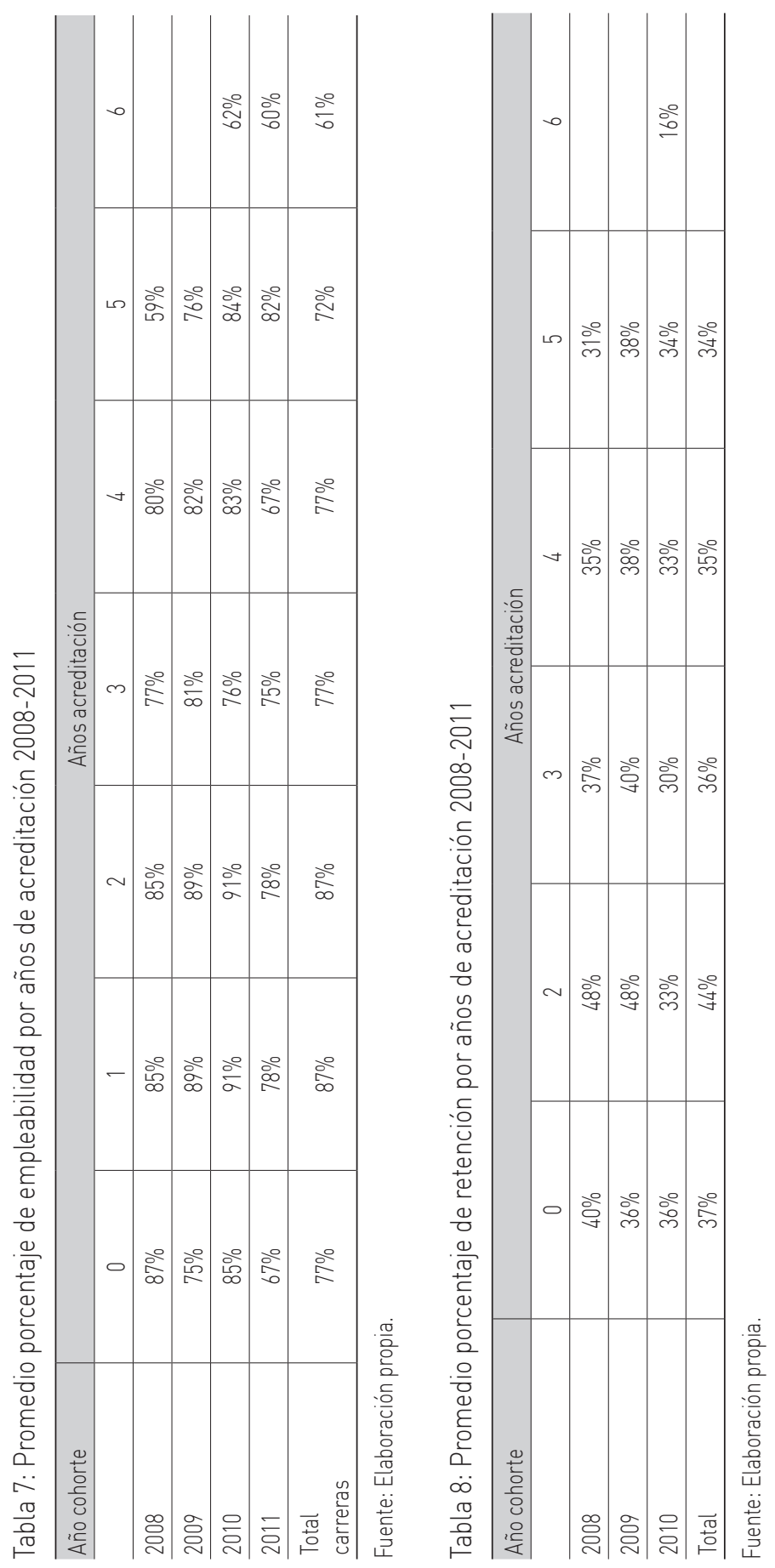




\subsection{Fase 2: Análisis de varianza}

Se presentan los resultados de los análisis ANOVA que muestran la asociación entre características institucionales y de las carreras con los indicadores de empleabilidad y retención.

\subsubsection{Variables a nivel de institución}

En primer lugar, se estudió la proporción de la varianza de los indicadores a nivel de carrera que se explica por la pertenencia a las distintas instituciones. La Tabla 9 muestra que la proporción de varianza del indicador de empleabilidad, atribuible a la pertenencia a una institución, se encuentra en un rango de $27 \%$ a $40 \%$. A su vez, la varianza explicada por diferencias en empleabilidad al interior de las instituciones -entre carreras de una misma institución- es mayor y se sitúa en el rango entre el $60 \%$ y el $73 \%$. Para el indicador de retención la tendencia es similar, sin embargo, la proporción de varianza explicada por la pertenencia a una institución es menor (20\%). La varianza entre carreras de una misma institución es más alta, ya que esta explica entre un $60 \%$ y $73 \%$ de la varianza de la retención. De lo anterior se deduce que, a medida que pasan los años, las características de la institución tienen menos importancia para explicar la empleabilidad a nivel de carrera; y que en ambos indicadores hay mayor varianza entre carreras de una misma institución que entre instituciones.

Tabla 9: Análisis de varianza según institución

\begin{tabular}{l|l|c|c|c|c}
\hline & & 2008 & 2009 & 2010 & 2011 \\
\hline \multirow{2}{*}{ Indicador empleabilidad } & Entre grupos & $33 \%$ & $40 \%$ & $40 \%$ & $27 \%$ \\
\cline { 2 - 6 } & Dentro grupos & $67 \%$ & $60 \%$ & $60 \%$ & $73 \%$ \\
\hline \multirow{2}{*}{ Indicador retención } & Entre grupos & $23 \%$ & $23 \%$ & $21 \%$ & \\
\cline { 2 - 6 } & Dentro grupos & $77 \%$ & $77 \%$ & $79 \%$ & \\
\hline
\end{tabular}

Fuente: Elaboración propia.

Sobre la base de estos resultados se estudió la descomposición de la varianza de los indicadores de empleabilidad y retención según características de las instituciones. En la Tabla 10 se presentan los resultados al descomponer la varianza de los indicadores en las variables: tipo de institución, años de acreditación, puntaje INICIA 
disciplinaria y puntaje PSU ingreso). Los resultados muestran que las diferencias entre los tipos de instituciones (privadas, universidades pertenecientes al CRUCH, universidades privadas del CRUCH, institutos profesionales) explican en un rango de 3\% a 16\% la varianza del indicador de empleabilidad, y en menor medida (máximo 6\%), la variación del indicador de retención. En otras palabras, las diferencias observadas en los indicadores de empleabilidad y retención de las distintas carreras se asocian levemente al tipo de institución a la cual pertenecen.

La segunda variable estudiada corresponde a los años de acreditación. Los resultados muestran que más del 5\% de la varianza del indicador de empleabilidad se explica por la pertenencia a instituciones con distintos años de acreditación (a excepción del año 2011 donde la proporción es menor); la cifra es menor para la retención. Este resultado muestra que la acreditación se vincula con la variación en tasas de empleabilidad y retención de las carreras, pero que esta asociación es menor a la explicada por la pertenencia a una institución.

Las últimas dos variables institucionales estudiadas, resultados en la prueba INCIA disciplinaria (años 2008 a 2010) y puntaje de corte en la PSU, muestran que el porcentaje explicado por la pertenencia a uno de los cuatro grupos de desempeño en la prueba INICIA (cuartiles) revela escasamente la varianza de ambos indicadores, esto es así en los cuatro años estudiados, siendo la mayor proporción la observada en el año 2009 en el indicador de empleabilidad (4\%).

En el caso del puntaje de corte de la PSU promedio lenguaje y matemática se construyó una variable que distingue tres grupos de carreras (aquellas que no registran puntaje de corte, puntaje de corte promedio lenguaje y matemática inferior a 500 y puntaje promedio superior a 500). De la Tabla 10 se desprende que alrededor de un 5\% de la varianza del indicador de empleabilidad se asocia a los puntajes de corte de la institución de proveniencia. Este efecto, prácticamente desaparece cuando se estudia la varianza del indicador de retención. 
Tabla 10: Análisis de varianza según variables institucionales 2008-2011

\begin{tabular}{|c|c|c|c|c|c|c|}
\hline \multirow[b]{2}{*}{ Variable } & \multirow[b]{2}{*}{ Indicador } & & \multicolumn{4}{|c|}{ Año cohorte } \\
\hline & & & 2008 & 2009 & 2010 & 2011 \\
\hline \multirow{4}{*}{ Tipo institución } & \multirow{2}{*}{ Empleabilidad } & Entre grupos & $6 \%$ & $16 \%$ & $11 \%$ & $3 \%$ \\
\hline & & Dentro grupos & $94 \%$ & $84 \%$ & $89 \%$ & $97 \%$ \\
\hline & \multirow{2}{*}{ Retención } & Entre grupos & $2 \%$ & $6 \%$ & $3 \%$ & \\
\hline & & Dentro grupos & $98 \%$ & $94 \%$ & $97 \%$ & \\
\hline \multirow{4}{*}{ Años acreditación } & \multirow{2}{*}{ Empleabilidad } & Entre grupos & $8 \%$ & $6 \%$ & $9 \%$ & $4 \%$ \\
\hline & & Dentro grupos & $92 \%$ & $94 \%$ & $91 \%$ & $96 \%$ \\
\hline & \multirow{2}{*}{ Retención } & Entre grupos & $7 \%$ & $3 \%$ & $2 \%$ & \\
\hline & & Dentro grupos & $93 \%$ & $97 \%$ & $98 \%$ & \\
\hline \multirow{4}{*}{$\begin{array}{l}\text { Puntaje INICIA } \\
\text { disciplinaria }\end{array}$} & \multirow{2}{*}{ Empleabilidad } & Entre grupos & $1 \%$ & $4 \%$ & $3 \%$ & $1 \%$ \\
\hline & & Dentro grupos & $99 \%$ & $96 \%$ & $97 \%$ & $99 \%$ \\
\hline & \multirow{2}{*}{ Retención } & Entre grupos & $3 \%$ & $2 \%$ & $2 \%$ & \\
\hline & & Dentro grupos & $97 \%$ & $98 \%$ & $98 \%$ & \\
\hline \multirow{4}{*}{$\begin{array}{l}\text { Puntaje PSU } \\
\text { ingreso }\end{array}$} & \multirow{2}{*}{ Empleabilidad } & Entre grupos & $2 \%$ & $7 \%$ & $5 \%$ & $2 \%$ \\
\hline & & Dentro grupos & $98 \%$ & $93 \%$ & $95 \%$ & $98 \%$ \\
\hline & \multirow{2}{*}{ Retención } & Entre grupos & $2 \%$ & $0 \%$ & $0 \%$ & \\
\hline & & Dentro grupos & $98 \%$ & $100 \%$ & $100 \%$ & \\
\hline
\end{tabular}

Fuente: Elaboración propia.

\subsubsection{Variables a nivel de carrera}

Los resultados anteriores sugieren que la variabilidad de los indicadores es mayor entre las distintas instituciones que entre las variables institucionales estudiadas. Por esto, se analizó la varianza de los indicadores de empleabilidad y retención considerando características de las carreras que varían al interior de las instituciones (región, horario en que se imparten y duración). Para ello, se estimó el promedio de la varianza entre grupos, entre carreras de una misma institución y el promedio de varianza dentro de los grupos dentro de la institución. La Tabla 11 muestra los resultados de estos análisis.

Respecto de las variables de las carreras se observa que la región en que se imparte explica una alta proporción de la varianza de ambos indicadores (empleabilidad y retención), en un rango que va desde un $31 \%$ a un $42 \%$. La descomposición de la varianza en tipos de horario muestra que el tipo de jornada (diurna, vespertina o a distancia) se 
42 INDICADORES DE RESULTADOS DE LAS PROGRAMAS DE PEDAGOGÍA BÁSICA: EVIDENCIA PARA MEJORAR EL ASEGURAMIENTO DE LA CALIDAD DE LOS DOCENTES EN CHILE - R. Rivero y C. Hurtado

asocia a la varianza de la empleabilidad entre carreras de una misma institución, esta proporción varía entre un $8 \%$ a un 11\%, según años. En el caso de la retención, el horario de la carrera explica desde un $6 \%$ a un $15 \%$ de la varianza del indicador.

Se consideran dos grupos de carreras en términos de duración, aquellas que se extienden por un máximo cuatro años (ocho semestres o menos) y aquellas que duran más que esto. Los resultados señalan que esta característica se relaciona con la varianza de empleabilidad en un rango que varía desde un 3\% a un 16\%, según año. En el caso de la retención los resultados muestran que, en promedio, la duración de las carreras explica alrededor de un 7\% de la varianza del indicador entre carreras de una misma institución.

Tabla 11: Análisis de varianza según variables de las carreras 2008-2011

\begin{tabular}{|c|c|c|c|c|c|c|}
\hline \multirow[b]{2}{*}{ Variable } & \multirow[b]{2}{*}{ Indicador } & & \multicolumn{4}{|c|}{ Año cohorte } \\
\hline & & & 2008 & 2009 & 2010 & 2011 \\
\hline \multirow{4}{*}{ Región } & \multirow{2}{*}{ Empleabilidad } & Entre grupos & $20 \%$ & $27 \%$ & $31 \%$ & $42 \%$ \\
\hline & & Dentro grupos & $80 \%$ & $73 \%$ & $69 \%$ & $58 \%$ \\
\hline & \multirow{2}{*}{ Retención } & Entre grupos & $31 \%$ & $32 \%$ & $38 \%$ & \\
\hline & & Dentro grupos & $69 \%$ & $68 \%$ & $62 \%$ & \\
\hline \multirow{4}{*}{ Horario } & \multirow{2}{*}{ Empleabilidad } & Entre grupos & $6 \%$ & $9 \%$ & $8 \%$ & $11 \%$ \\
\hline & & Dentro grupos & $94 \%$ & $91 \%$ & $92 \%$ & $89 \%$ \\
\hline & \multirow{2}{*}{ Retención } & Entre grupos & $7 \%$ & $13 \%$ & $15 \%$ & \\
\hline & & Dentro grupos & $93 \%$ & $87 \%$ & $85 \%$ & \\
\hline \multirow{4}{*}{ Duración } & \multirow{2}{*}{ Empleabilidad } & Entre grupos & $12 \%$ & $3 \%$ & $6 \%$ & $16 \%$ \\
\hline & & Dentro grupos & $88 \%$ & $97 \%$ & $94 \%$ & $84 \%$ \\
\hline & \multirow{2}{*}{ Retención } & Entre grupos & $6 \%$ & $9 \%$ & $7 \%$ & \\
\hline & & Dentro grupos & $94 \%$ & $91 \%$ & $93 \%$ & \\
\hline
\end{tabular}

Fuente: Elaboración propia.

Otro factor que podría incidir en la empleabilidad es el año de titulación, para explorar en esta variable se realizó un análisis ANOVA considerando el año de la cohorte; en la Tabla 12 se exponen los resultados. El año de titulación incide de manera similar en la varianza de ambos indicadores (3\%); esto indicaría que ambas variables son relativamente estables a lo largo de las cohortes consideradas en el estudio. 
Tabla 12: Análisis de varianza según año

\begin{tabular}{l|l|c}
\hline & & Año \\
\hline \multirow{3}{*}{ Indicador empleabilidad } & Entre grupos & $3 \%$ \\
\cline { 2 - 3 } & Dentro grupos & $97 \%$ \\
\hline \multirow{2}{*}{ Indicador retención } & Entre grupos & $3 \%$ \\
\cline { 2 - 3 } & Dentro grupos & $97 \%$ \\
\hline
\end{tabular}

Fuente: Elaboración propia.

\subsection{Fase 3: Modelos}

Tras estos resultados se estimaron modelos lineales jerárquicos cuyas variables dependientes corresponden a los indicadores de empleabilidad y retención. Debido a la importancia de las instituciones según los análisis ANOVA se optó por usar todas las carreras con datos disponibles (774) y anidar sus resultados en la institución de pertenencia. Luego se incluyeron como covariables las características institucionales, las de la carrera y las contextuales (región y año). Las Tablas 13 y 14 muestran los resultados de los modelos que tienen como variable dependiente la empleabilidad y retención, respectivamente. En ambos casos, los modelos 1 a 3 consideran solo la variable años de acreditación de la carrera como proxy de la calidad de la formación. En los modelos 4 a 6 se suma la variable promedio de INICIA disciplinaria. Se considera que las variables acreditación e INICIA son indicativas de la calidad de las instituciones y, por lo tanto, se busca estudiar su asociación con los indicadores de empleabilidad y retención ${ }^{21}$.

En términos de la empleabilidad, se observa que los años de acreditación se asocian de manera significativa a la tasa de empleabilidad solo en los modelos en que se controla por el puntaje INICIA y por la región y año. Los resultados señalan que carreras de instituciones con dos años de acreditación presentan mayores tasas de empleabilidad que aquellas a las que se les rechazó la acreditación. La categoría tres años de acreditación resulta significativamente relacionada con una mayor empleabilidad solo en modelo 6. El resto de las categorías de años de acreditación no se asocia significativamente a

21 La correlación entre ambas es de 0,47 y es significativa a un nivel de confianza de 0,05. 
ninguna de las especificaciones del modelo. Por su parte, el puntaje en la prueba INICIA no se relaciona significativamente con el indicador de empleabilidad en ninguno de los modelos.

En relación con otras características institucionales, los resultados muestran que el tipo de institución se asocia significativamente con la empleabilidad. Así carreras de instituciones privadas y estatales pertenecientes al CRUCH presentan consistentemente una mayor empleabilidad al compararlas con carreras de institutos profesionales. El puntaje de corte en la PSU de ingreso también se asocia significativamente con la tasa de empleabilidad, de tal forma que aquellas con puntajes de corte iguales o inferiores a 500 presentan mayores valores en el indicador si se comparan con aquellas que no solicitan puntajes en la prueba de selección. En los modelos 3 y 6 , la categoría de "más de 500 puntos" presenta una asociación positiva y significativa, al comparar con carreras sin requisitos de puntaje.

Los resultados de las variables de las carrera muestran que el horario diurno se asocia significativa y positivamente al indicador si se lo compara con el horario vespertino, sin embargo, dicha significancia se pierde al incorporar la variable INICIA. Respecto de la duración, más semestres se relacionan significativamente con mayor empleabilidad. Al incorporar la región y el año (modelos 3 y 6) se observan coeficientes negativos y significativos para algunas regiones (segunda, cuarta y sexta). Lo mismo ocurre con uno de los años de titulación (2011), que presenta un coeficiente negativo y significativo al comparar con el año 2008. Adicionalmente, en el modelo 3 -donde no se controla por el puntaje promedio de la institución en la prueba INICIA disciplinaria- se obtiene que el año 2010 se relaciona positiva y significativamente con mayores tasas de empleabilidad si se lo compara con el año 2008.

Los resultados para el indicador retención (Tabla 14) muestran, en general, una pérdida de relevancia de las variables institucionales. En cuanto al tipo de institución, solo las privadas pertenecientes al CRUCH muestran una relación significativa, y negativa, con la retención al compararlas con los institutos profesionales. Esto sucede en los modelos 3 y 6 , que integran como covariable el puntaje INICIA. 
Luego las carreras más selectivas, en términos de la PSU, presentan significativamente mayores tasas de retención al compararlas con aquellas que no exigen puntajes en esta prueba, efecto solo observable en el modelo 5.

Sobre las variables que difieren entre carreras de una misma institución, la jornada en que se imparte la carrera no es significativa y tampoco lo es la duración. La prueba INICIA no resulta significativa como predictor de retención.

Por último, al incluir las variables región y año, se obtiene que solo una región resulta significativamente relacionada con la retención, y en un sentido negativo, en los modelos 3 y 6 (cuarta región). Al controlar por el resultado institucional en la prueba INICIA se observa que las regiones quinta, octava y décima se relacionan, significativamente, a menor retención. También, las carreras de la cohorte 2010 presentan significativamente menor retención que las del 2008. Como se mencionó anteriormente esto podría explicarse por la menor disponibilidad de los datos para construir la variable de retención para este año al compararla con los años 2008 y 2009.

En síntesis los resultados de la fase 3 son coherentes con los obtenidos en la fase anterior del estudio, ya que las variables institucionales que mostraron mayor relación con la varianza de los indicadores mantienen significancia (tipo de institución y selectividad), pese a las distintas especificaciones de las regresiones. Estas relaciones son consistentes a diferencia de las observadas con las variables de carrera y se encuentran en mayor medida asociadas a la empleabilidad que a la retención. Los años de acreditación aparecen débilmente asociados a la empleabilidad y retención de los egresados. 
46 INDICADORES DE RESULTADOS DE LAS PROGRAMAS DE PEDAGOGÍA BÁSICA: EVIDENCIA PARA MEJORAR EL ASEGURAMIENTO DE LA CALIDAD DE LOS DOCENTES EN CHILE - R. Rivero y C. Hurtado

Tabla 13: Modelos indicador empleabilidad

\begin{tabular}{|c|c|c|c|c|c|c|}
\hline VARIABLES & 1 & 2 & $3^{*}$ & 4 & 5 & $6^{*}$ \\
\hline Dos años ac. & 0,0528 & 0,0459 & 0,0831 & 0,0978 & $0,165^{* * *}$ & $0,160 * * *$ \\
\hline Ref: sin ac. & $(0,0640)$ & $(0,0617)$ & $(0,0553)$ & $(0,0616)$ & $(0,0511)$ & $(0,0373)$ \\
\hline \multirow[t]{2}{*}{ Tres años ac. } & $-0,0832$ & $-0,0766$ & $-0,0438$ & 0,00632 & 0,0766 & $0,0648 *$ \\
\hline & $(0,0517)$ & $(0,0510)$ & $(0,0471)$ & $(0,0547)$ & $(0,0498)$ & $(0,0393)$ \\
\hline \multirow[t]{2}{*}{ Cuatro años ac. } & $-0,0447$ & $-0,0356$ & $-0,0459$ & 0,00119 & 0,0576 & 0,0342 \\
\hline & $(0,0504)$ & $(0,0500)$ & $(0,0463)$ & $(0,0544)$ & $(0,0493)$ & $(0,0408)$ \\
\hline \multirow[t]{2}{*}{ Cinco años ac. } & $-0,106$ & $-0,0660$ & $-0,0674$ & $-0,0116$ & 0,0736 & 0,0637 \\
\hline & $(0,0738)$ & $(0,0750)$ & $(0,0702)$ & $(0,0783)$ & $(0,0705)$ & $(0,0655)$ \\
\hline \multirow[t]{2}{*}{ Seis años ac. } & $-0,0491$ & $-0,0281$ & $-0,0285$ & $-0,109$ & $-0,0469$ & 0,0465 \\
\hline & $(0,116)$ & $(0,120)$ & $(0,127)$ & $(0,113)$ & $(0,108)$ & $(0,109)$ \\
\hline \multirow[t]{2}{*}{ INICIA } & & & & 0,204 & 0,169 & 0,122 \\
\hline & & & & $(0,145)$ & $(0,146)$ & $(0,143)$ \\
\hline U. privadas & & $-0,00443$ & $0,129 * *$ & & $0,198^{* * *}$ & $0,284^{* * *}$ \\
\hline Ref: I. profesionales & & $(0,0663)$ & $(0,0654)$ & & $(0,0653)$ & $(0,0555)$ \\
\hline \multirow[t]{2}{*}{ U. estatales CRUCH } & & $0,0809 *$ & $0,142^{* * *}$ & & $0,102^{* * *}$ & $0,130^{* * *}$ \\
\hline & & $(0,0425)$ & $(0,0405)$ & & $(0,0310)$ & $(0,0236)$ \\
\hline \multirow[t]{2}{*}{ U. privadas $\mathrm{CRUCH}$} & & -0.0495 & $-0,0394$ & & $-0,00683$ & $-0,0467$ \\
\hline & & $(0,0595)$ & $(0,0563)$ & & $(0,0464)$ & $(0,0407)$ \\
\hline Menos o igual 500 ptos. & & 0,0429 & $0,0728^{* *}$ & & $0,0723^{* *}$ & $0,103^{* * *}$ \\
\hline ref: no requiere ptje. PSU & & $(0,0326)$ & $(0,0313)$ & & $(0,0349)$ & $(0,0298)$ \\
\hline \multirow[t]{2}{*}{ Más de 500 ptos. } & & 0,0182 & $0,0833^{* * *}$ & & 0,0508 & $0,0954^{* *}$ \\
\hline & & $(0,0319)$ & $(0,0323)$ & & $(0,0408)$ & $(0,0373)$ \\
\hline Diurno & & & $0,0498 * * *$ & & & $0,0427 * *$ \\
\hline Ref: Vespertino & & & $(0,0187)$ & & & $(0,0199)$ \\
\hline \multirow[t]{2}{*}{ A distancia } & & & 0,00574 & & & 0,0451 \\
\hline & & & $(0,0877)$ & & & $(0,113)$ \\
\hline \multirow[t]{2}{*}{ No semestres } & & & $0,0243^{* * *}$ & & & $0,0223^{* *}$ \\
\hline & & & $(0,00810)$ & & & $(0,00984)$ \\
\hline \multirow[t]{2}{*}{ Constante } & $0,818^{* * *}$ & $0,753^{* * *}$ & $0,480 * * *$ & $0,673^{* * *}$ & $0,522 * * *$ & $0,347^{* * *}$ \\
\hline & $(0,0398)$ & $(0,0592)$ & $(0,0979)$ & $(0,0756)$ & $(0,0768)$ & $(0,121)$ \\
\hline Observaciones & 733 & 733 & 697 & 552 & 552 & 537 \\
\hline Número de grupos & 49 & 49 & 49 & 40 & 40 & 40 \\
\hline
\end{tabular}

Standard errors in parentheses

Nota $\left({ }^{*}\right)$ : En este modelo se incluyeron las variables región y año. ${ }^{* * *} p<0,01 ;{ }^{* *} p<0,05 ;{ }^{*} p<0,1$ 
Tabla 14: Modelos indicador retención

\begin{tabular}{|c|c|c|c|c|c|c|}
\hline VARIABLES & 1 & 2 & $3^{*}$ & 4 & 5 & $6^{*}$ \\
\hline Dos años ac. & 0,0480 & 0,0493 & 0,0521 & 0,0593 & 0,0692 & 0,0544 \\
\hline Ref: sin ac. & $(0,0408)$ & $(0,0428)$ & $(0,0394)$ & $(0,0507)$ & $(0,0664)$ & $(0,0619)$ \\
\hline \multirow[t]{2}{*}{ Tres años ac. } & $-0,0344$ & $-0,0247$ & $-0,0142$ & $-0,0234$ & $-0,00575$ & $-0,00340$ \\
\hline & $(0,0373)$ & $(0,0402)$ & $(0,0397)$ & $(0,0501)$ & $(0,0659)$ & $(0,0612)$ \\
\hline \multirow[t]{2}{*}{ Cuatro años ac. } & $-0,0273$ & $-0,0216$ & $-0,0279$ & $-0,0390$ & $-0,0422$ & $-0,0534$ \\
\hline & $(0,0386)$ & $(0,0416)$ & $(0,0403)$ & $(0,0540)$ & $(0,0654)$ & $(0,0615)$ \\
\hline \multirow[t]{2}{*}{ Cinco años ac. } & $-0,0407$ & $-0,0283$ & $-0,0165$ & 0,00497 & $-0,0296$ & $-0,0929$ \\
\hline & $(0,0559)$ & $(0,0621)$ & $(0,0641)$ & $(0,0739)$ & $(0,0853)$ & $(0,0878)$ \\
\hline \multirow[t]{2}{*}{ Seis años ac. } & $-0,214$ & $-0,145$ & $-0,0184$ & $-0,230$ & $-0,138$ & 0,0238 \\
\hline & $(0,137)$ & $(0,144)$ & $(0,167)$ & $(0,141)$ & $(0,153)$ & $(0,171)$ \\
\hline \multirow[t]{2}{*}{ INICIA } & & & & $-0,0226$ & $-0,0349$ & 0,0791 \\
\hline & & & & $(0,159)$ & $(0,174)$ & $(0,183)$ \\
\hline U. privadas & & $-0,0156$ & $-0,0146$ & & 0,0269 & 0,00859 \\
\hline Ref: I. profesionales & & $(0,0521)$ & $(0,0591)$ & & $(0,0881)$ & $(0,0853)$ \\
\hline \multirow[t]{2}{*}{ U. estatales CRUCH } & & 0,0357 & 0,0202 & & 0,0383 & 0,0192 \\
\hline & & $(0,0318)$ & $(0,0324)$ & & $(0,0322)$ & $(0,0320)$ \\
\hline \multirow[t]{2}{*}{ U. privadas CRUCH } & & $-0,0581$ & $-0,0905 *$ & & $-0,0535$ & $-0,114^{* *}$ \\
\hline & & $(0,0481)$ & $(0,0504)$ & & $(0,0505)$ & $(0,0541)$ \\
\hline Menos o igual 500 ptos. & & 0,0131 & $-0,00145$ & & 0,00837 & $-0,0247$ \\
\hline Ref: no requiere ptje. PSU & & $(0,0329)$ & $(0,0331)$ & & $(0,0407)$ & $(0,0395)$ \\
\hline \multirow[t]{2}{*}{ Más de 500 ptos. } & & 0,0440 & 0,0485 & & $0,101^{*}$ & 0,0703 \\
\hline & & $(0,0346)$ & $(0,0371)$ & & $(0,0514)$ & $(0,0508)$ \\
\hline Diurno & & & $-0,0194$ & & & $-0,0268$ \\
\hline Ref: Vespertino & & & $(0,0234)$ & & & $(0,0271)$ \\
\hline \multirow[t]{2}{*}{ A distancia } & & & 0,0718 & & & 0,0760 \\
\hline & & & $(0,105)$ & & & $(0,158)$ \\
\hline $\mathrm{N}^{0}$ semestres & & & $-0,00663$ & & & 0,0120 \\
\hline \multirow[t]{2}{*}{ Constante } & $0,378^{* * *}$ & $0,344^{* * *}$ & $0,484^{* * *}$ & $0,397 * * *$ & $0,355^{* * *}$ & $0,342^{* *}$ \\
\hline & $(0,0292)$ & $(0,0472)$ & $(0,108)$ & $(0,0829)$ & $(0,1000)$ & $(0,166)$ \\
\hline Observaciones & 553 & 553 & 525 & 408 & 408 & 394 \\
\hline Número de grupos & 48 & 48 & 48 & 38 & 38 & 38 \\
\hline
\end{tabular}

Standard errors in parentheses

Nota $\left({ }^{*}\right)$ : en este modelo se incluyeron las variables región y año. ${ }^{* *} p<0,01 ;{ }^{* *} p<0,05 ;{ }^{*} p<0$. 


\section{Conclusiones}

En este estudio se explora la construcción de indicadores de empleabilidad de Pedagogía Básica, específicamente, empleabilidad durante los primeros tres años a partir de la titulación y retención entre el cuarto y sexto año tras el egreso. Un primer hallazgo son los bajos niveles de empleabilidad y retención a nivel de institución y carrera. Bajo la metodología usada, la tasa de empleabilidad promedio es de $80 \%$ y solo un quinto de los egresados se mantiene trabajando en el sistema escolar a cuatro, cinco o seis años de su titulación. En un $3 \%$ de las carreras entre 2008 y 2011 , ninguno de sus egresados se empleó en el sistema escolar tras titularse y en $6 \%$ de estas, ninguno continúa trabajando entre el cuarto y sexto año.

En segundo lugar, pese a que en promedio la empleabilidad y retención son similares a nivel de institución y carrera, se encuentra una alta varianza a nivel de ambas unidades, mayor entre carreras de una misma institución (alrededor de un 70\% de la varianza de ambos indicadores se explica por diferencias dentro de las instituciones) que entre instituciones (alrededor de un 30\% de la varianza se explica por diferencias entre instituciones).

En tercer lugar, respecto de las características institucionales y de las carreras, el estudio de descomposición de varianza muestra que las características institucionales estudiadas -tipo de institución, años de acreditación, selectividad al ingreso y puntaje en INICIAse asocian a la varianza de la empleabilidad en mayor medida que a la retención. Es el tipo de institución la característica mayormente relacionada con los indicadores. Por su parte la región, horario y duración en semestres (características de las carreras, que varían a nivel de institución) explican parte de la varianza de los indicadores, siendo la más relevante la región donde se imparte la carrera. Los resultados de los modelos lineales jerárquicos confirman la asociación entre características institucionales y de las carreras con la empleabilidad y la menor asociación con la retención. En particular, la acreditación resulta un indicador débil y no consistentemente asociado con la empleabilidad de las carreras y, a su vez, no relacionado con la retención. Por su parte, los resultados institucionales en la prueba 
INICIA no se relacionan con la empleabilidad ni con la retención de los titulados de carreras de Pedagogía Básica.

La interpretación de estos resultados requiere considerar que la empleabilidad de los docentes novatos depende de factores dinámicos de la oferta (docentes) y de la demanda (establecimientos), siendo difícil identificar cuál de ellos es más determinante en las trayectorias laborales (Hanushek y Rivkin, 2006). En el ámbito de la oferta laboral, además de las cualidades personales de los docentes o de las características de sus programas de formación, inciden la proporción de quienes ingresan al sistema escolar, stock acumulado de profesores y tasas de retiro (Sánchez et al., 2013). Por su parte, la demanda laboral está influenciada por la matrícula de los alumnos, razón de alumnos por profesor, necesidades de profesores con especialidad y tasas de contratación por tipo de establecimiento y ubicación geográfica (Sánchez, Gutiérrez, Hochschild, Medeiros, Ortiz y Sepúlveda, 2013). A esto se añade la complejidad propia del sistema chileno, cuya alta atomización por tipos de dependencia define distintos sistemas de contratación; hasta ahora, los requisitos para ingresar al sistema municipal son definidos por el Estatuto Docente y por los empleadores en el sector privado (Flores et al., 2013). De esta manera, la baja asociación entre la acreditación y la empleabilidad y retención, al igual que con los resultados en la prueba INICIA, puede deberse a que las escuelas no consideran esta información para emplear a los docentes, o bien, a que la oferta se concentra en escuelas con determinadas características asociadas a la empleabilidad o a la retención. También puede explicarse porque la acreditación no incluye características que potencien la inserción de los docentes y su retención en las escuelas. En cualquier caso, será relevante continuar explorando en características de los programas que se asocien positivamente a la empleabilidad y retención, asunto en el que este estudio ha avanzado.

Estos resultados alertan, primero, acerca de la importancia de monitorear las tasas de empleabilidad y retención alcanzadas por las instituciones y carreras que imparten Pedagogía Básica en Chile. Debido a que la metodología usada es poco exigente, ya que solo se considera que los titulados se emplean o mantienen en el sistema si 
aparecen en uno de los años de las bases ministeriales, es posible que las tasas de empleabilidad y retención estén sobreestimadas. Otra limitación es que se desconoce si los egresados que no aparecen en las bases de los establecimientos se desempeñan como profesores en otro ámbito, ya que si este efecto es amplio, los indicadores estarían subestimados. En segundo lugar, la alta varianza entre carreras de una misma institución sugiere que es importante estudiar qué unidad es más adecuada para el proceso de acreditación, que hasta ahora se realiza a nivel de instituciones y no de carreras. Esto es particularmente relevante en un contexto de cambios al sistema de aseguramiento de la calidad de la educación superior y cambios a la carrera docente.

La revisión de otros sistemas educacionales orienta respecto de alternativas para integrar la empleabilidad y la retención en la acreditación; ya sea en el ámbito de los resultados (evaluando las tasas de empleabilidad) ${ }^{22}$ o en el ámbito de los procesos (evaluando sistemas de seguimiento de los egresados y evaluación de empleadores). Para la acreditación de TEAC (Estados Unidos) los programas deben demostrar tanto la capacidad de producir esta información como de usarla para la toma de decisiones orientadas a la mejora de la formación.

\section{Referencias}

Boyd, D., Lankford, H., Loeb, S., \& Wyckoff, J. (2003). Analyzing the determinants of the matching public school teachers to jobs: Estimating compensating differentials in imperfect labor markets (No. w9878). National Bureau of Economic Research. http://dx.doi.org/10.3386/w9878

Boyd, D., Lankford, H., Loeb, S., \& Wyckoff, J. (2005). The draw of home: How teachers' preferences for proximity disadvantage urban schools. Journal of Policy Analysis and Management, 24(1), 113-132. http://dx.doi. org/10.1002/pam.20072

Cabezas, V., Gallego, F., Santelices, V., y Zarhi, M. (2011). Factores correlacionados con las trayectorias laborales de docentes en Chile,

22 En este caso se advierte que la empleabilidad es un indicador que debe interpretarse cuidadosamente, ya que en un contexto de escasez de docentes no se relacionaría con la calidad de los programas. Del mismo modo, el porcentaje de candidatos que superan los exámenes de egreso es información poco relevante en un contexto en donde la mayoría de ellos lo logra (TEAC, 2012a). 
con especial énfasis en sus atributos académicos. Proyecto FONIDE N FS511082-2010. Santiago: Ministerio de Educación.

Centro de Estudios de Políticas y Prácticas en Educación, CEPPE (2013). Servicio de asesoría en Formación Inicial Docente (ID: 307-4-L113).

Council for the Accreditation of Educator Preparation, CAEP (2013). Accreditation Standards and Recommendations. Recuperado de http:// caepnet.org/standards/introduction

Council for Higher Education Accreditation (2010). The value of Accreditation, Recuperado de http://www.chea.org/pdf/Value\%20of\%20US\%20 Accreditation\%2006.29.2010_buttons.pdf

Cox, C., Meckes, L., y Bascopé, M. (2011). La institucionalidad formadora de profesores en Chile en la década del 2000: velocidad del mercado y parsimonia de las políticas. Pensamiento Educativo, 46-47, 205-245.

Consejo Nacional de Educación. INDICES Matrícula pregrado-postgrado, años 2005-2015. Recuperado de http://www.cned.cl/public/secciones/ SeccionIndicesPostulantes/Indices_Sistema.as

Comisión Nacional de Acreditación. Criterios de evaluación de carreras de educación. Recuperado de https://www.cnachile.cl/Criterios\%20 de\%20carreras/CriteriosEducacionFINAL1.pdf

Department for Education. (2011). Teacher's standards. Recuperado de https:// www.gov.uk/government/uploads/system/uploads/attachment_data/ file/283566/Teachers_standard_information.pdf

Domínguez, M., Bascopé, M., Meckes, L., y San Martín, E. (2012). ¿Producen mejores resultados las carreras de Pedagogía Básica con más años de acreditación? Estudios Públicos, 128, 1ه59.

Domínguez, M., y Meckes, L. (2011). Análisis y propuestas para la acreditación de pedagogías en Chile. Calidad en la Educación, (n 34), 165-183. Recuperado de http://dx.doi.org/10.4067/S0718-45652012000100002

Domínguez, M., y Meckes. L. (2012). Procesos de acreditación de Pedagogías: un estudio del quehacer de las agencias. Informe Final. Consejo Superior de Educación.

Evaluación INICIA (2014). Recuperado de http://www.mineduc.cl/index. php?id_portal=79

Eyzaguirre, S., e Inostroza, D. (2014). Antecedentes para perfeccionar la formación inicial docente. Puntos de Referencia, no 379 Recuperado de http://www.cepchile.cl/dms/archivo_5654_3597/pder379_ SEyzaguirre-DInostroza.pdf. 
52 INDICADORES DE RESULTADOS DE LAS PROGRAMAS DE PEDAGOGÍA BÁSICA: EVIDENCIA PARA MEJORAR EL ASEGURAMIENTO DE LA CALIDAD DE LOS DOCENTES EN CHILE - R. Rivero y C. Hurtado

Flores, C., Ortúzar, S., Ayala, P., y Milesi, C. (2013). Buscando la aguja en el pajar: proceso de búsqueda y contratación de profesores en la Región Metropolitana. Centro de estudios Mineduc. Estudios de política educativa, 1(1), 19-58. Recuperado de http://centroestudios.mineduc. cl/tp_enlaces/portales/tp5996f8b7cm96/uploadImg/File/Revista/ Revista_Estudios_Politica_Educativa_PDFFINAL.pdf

Freedman, S., \& Appleman, D. (2009). In it for the long haul: How teacher education can contribute to teacher retention in high-poverty, urban schools. Journal of Teacher Education, 3(60), 323-337. http://dx.doi. org/10.1177/0022487109336181

Hanushek, E., \& Rivkin, S., (2006). School quality and the black-white achievement gap, NBER Working Papers,12.651, National Bureau of Economic Research, Inc. Recuperado de http://dx.doi.org/10.3386/ w12651

Ingersoll, R. M., \& Strong, M. (2011). The impact of induction and mentoring programs for beginning teachers a critical review of the research. Review of educational research, 81(2), 201-233. Recuperado de http://dx.doi. org/10.3102/0034654311403323

Ingvarson, L. (2013a). Estándares de egreso y certificación inicial docente: la experiencia internacional. Calidad en la educación, 38, 22-77. http:// dx.doi.org/10.4067/S0718-45652013000100010

Ingvarson, L. (2013b). Quality assurance and teacher education: Summary of findings. En: L. Ingvarson et al. (Eds.), An analysis of teacher education context, Structure, and quality assurance. Arragements in TEDS - M Countries (pp. 227-251). Amsterdam: International Association for the Evaluation of Educational Achievement (IEA).

Ingvarson, L., Elliott, A., Kleinhenz, E., \& McKenzie, P. (2006). Teacher education accreditation: A review of national and international trends and practices. Teacher Education, 1. Recuperado de http://research.acer. edu.au/cgi/viewcontent.cgi?article=1000\&context=teacher_education

Ingvarson, L., Schwille, J., Rowley, G., Tatto, M., Senk, S., \& Peck, R. (2011). National policies and regulatory arrangements for the mathematics preparation of future teachers in sixteen countries.

Jorissen, K. T. (2003). Successful career transitions: Lessons from urban alternate route teachers who stayed. The High School Journal, 86(3), 41-51. http://dx.doi.org/10.1353/hsj.2003.0003

Kirchhoff, A., \& Lawrenz, F. (2011). The use of grounded theory to investigate the role of teacher Education on STEM teachers' career paths in highneed schools. Journal of Teacher Education, 62, 246-259. http://dx. doi. org/10.1177/0022487110397840 
Lemeitre, M. J. (2010). Aseguramiento de la calidad en Chile: impacto y proyecciones. Congreso retos y expectativas. Consejo Nacional de Educación, Conferencias. Recuperado de http://www.cned.cl/public/ secciones/seccionpublicaciones/publicaciones_seminarios_detalle. aspx?idPublicacion=51.

Ley $n^{\circ} 20.129$. Establece un sistema nacional de aseguramiento de la calidad de la educación superior. Publicada en el Diario Oficial el 17 de noviembre de 2006.

Liu, E., \& Johnson, S. (2006). New teachers' experiences of hiring: Late, rushed, and information-poor. Educational Administration Quarterly, 42, 324-360. http://dx.doi.org/10.1177/0013161X05282610

Maier, A., \& Youngs, P. (2009). Teacher preparation programs and teacher labor markets: How social capital may help explain teachers' career choices. Journal of Teacher Education, 60(4), 393-407. http://dx.doi. org/10.1177/0022487109341149

Meckes, L., \& Bascopé, M. (2010). Distribución inequitativa de los nuevos profesores mejor preparados. En Primer Congreso Interdisciplinario de Investigación en Educación, CIAE_CEPPE. Santiago, Chile.

Meckes, L., Taut, S., Bascopé, M., Valencia, E., \& Manzi, J. (2012). INICIA and the responses of teacher education institutions to increased accountability in Chile. Segundo Congreso Interdisciplinario de Investigación en Educación, Centro en Investigación Avanzada de la Universidad de Chile y Centro de Estudios de Políticas y Prácticas en Educación, Santiago, Chile.

Mizala, A., Hernández, T., y Makovec, M. (2013). Determinantes de la elección y deserción de la carrera de pedagogía. En Evidencias para Políticas Públicas en Educación. Selección de Investigaciones $5^{\circ}$ Concurso FONIDE (pp. 165-203). Santiago de Chile: Ministerio de Educación.

Mora, J. G. (2004). La necesidad del cambio educativo para la sociedad del conocimiento. Revista Iberoamericana de Educación, 35, 13-37.

Morse, J. A. (2006). The INQAAHE guidelines of good practice for external quality assurance agencies: assessment and next steps. Quality in Higher Education, 12, 243-252. http://dx.doi. org/10.1080/13538320601051051

National College for Teaching and Leadership, NCTL. (2014). Qualified teacher status (QTS): Qualify to teach in England. Recuperado de https://www. gov.uk/guidance/qualified-teacher-status-qts

Organisation for Economic Cooperation and Development., OECD (2005). Teachers matter: Attracting, developing and retaining effective teachers. Paris: OECD Publishing. 
54 INDICADORES DE RESULTADOS DE LAS PROGRAMAS DE PEDAGOGÍA BÁSICA: EVIDENCIA PARA MEJORAR EL ASEGURAMIENTO DE LA CALIDAD DE LOS DOCENTES EN CHILE - R. Rivero y C. Hurtado

Ministerio de Educación de Chile, Mineduc. (2010). Para una Educación, P. D. E. de Calidad. Informe final: primera etapa, propuestas para fortalecer la profesión docente en el sistema escolar chileno. Santiago: Autor.

Paredes, R., Bogolasky, F., Cabezas, V., Rivero, R., y Zahri, M. (2013). Los determinantes del primer trabajo para profesores de educación básica en la Región Metropolitana. Fondo de Investigación y Desarrollo en Educación, Ministerio de Educación

Portal Mi Futuro (2015). Recuperado de http://www.mifuturo.cl/index.php/ futuro-laboral/buscador-por-carrera

Rivero, M. (2015). The link of teacher career paths on the distribution of high qualified teachers: A Chilean case study. Education policy analysis archives, 23.

Ruffinelli, A., y Guerrero, A. (2009). Círculo de segmentación del sistema educativo chileno: destino laboral de egresados de Pedagogía en Educación Básica. Calidad en la Educación, no 31, 19-44.

Sánchez, M. J., Gutiérrez, G., Hochschild, H., Medeiros, M. P., Ortiz, M., y Sepúlveda, M. J. (2013). Mercado de profesores en el sistema escolar urbano chileno. Calidad en la educación, 39, 156-194. Recuperado de http://www.scielo.cl/scielo.php?script=sci_arttext\&pid=S071845652013000200006\&lng=en\&nrm=iso\&tlng=es

Scharager, J., Cortés, F., y Bravo, T. (2008) Percepción de la calidad actual de los titulados y graduados de la educación superior chilena. Santiago de Chile: Centro de Medición de la Escuela de Psicología de la Pontificia Universidad Católica de Chile, financiado por Mineduc y BID.

Tatto, M. T., Peck, R., Schwille, J., Bankov, K., Senk, S. L., Rodríguez, M., \& Rowley, G. (2012). Policy, practice, and readiness to teach primary and secondary mathematics in 17 countries: Findings from the IEA.

Teacher Education Accreditation Council, TEAC. (2012a). Guide to the TEAC Audit. Recuperado de http://www.teac.org/wp-content/ uploads/2009/03/TEAC-Audit-Guide-2010.pdf

Teacher Education Accreditation Council, TEAC. (2012b). Guide to accreditation. Recuperado de http://www.teac.org/wp-content/ uploads/2012/01/GuideToAccred-for-2012-V1.12.pdf

The National Council for Accreditation of Teacher Education, NCATE. (20102014). PENDIENTE EL NOMBRE DEL DOCUMENTO UTILIZADO. Recuperado de http://www.ncate.org/Accreditation/tabid/100/Default. $\operatorname{aspx}$ 
Tuning Project. (2006). Una introducción a Tuning Educational Structures in Europe. La contribución de las universidades al proceso de Bolonia. Recuperado de http://www.unideusto.org/tuningeu/images/stories/ documents/General_Brochure_Spanish_version.pdf General_ Brochure_Spanish_version.pdf

Zapata, G., y Tejeda, I. (2009). Informe nacional Chile educación superior y mecanismos de aseguramiento de la calidad. Proyecto ALFA "Aseguramiento de la Calidad: Políticas Públicas y Gestión Universitaria”. Santiago: Centro Interuniversitario de Desarrollo, CINDA.

Recibido: 20/07/2015

Aceptado: 20/10/2015 